\title{
Selective Presynaptic Propagation of Long-Term Potentiation in Defined Neural Networks
}

\author{
Hui-zhong W. Tao, Li I. Zhang, Guo-qiang Bi, and Mu-ming Poo \\ Department of Biology, University of California at San Diego, La Jolla, California 92093-0357
}

Induction of long-term potentiation (LTP) of the synaptic connection between two hippocampal glutamatergic neurons in a neural network formed in cell culture resulted in a specific pattern of potentiation at other connections within the network. We found that potentiation propagated from the site of induction retrogradely to glutamatergic or GABAergic synapses received by the dendrites of the presynaptic neuron and laterally to those made by its axonal collaterals onto other glutamatergic cells. In contrast, synapses made by the same presynaptic neuron onto GABAergic cells were not affected, and there was no postsynaptic lateral or forward propagation to other syn- apses received or made by the postsynaptic neuron. In addition, there was no secondary propagation to synapses not directly associated with the presynaptic neuron. Both induction and propagation of LTP required correlated spiking of the postsynaptic cell as well as the activation of the NMDA subtype of glutamate receptors. Such selective propagation suggests the existence of a long-range cytoplasmic signaling within the presynaptic neuron, leading to a specific pattern of coordinated potentiation along excitatory pathways in a neural network.

Key words: synaptic plasticity; LTP; hippocampal culture; correlated activity; spike timing; Hebbian; synapse specificity
In search of a cellular basis of associative learning, Hebb (1949) suggested that repetitive correlated excitation of presynaptic and postsynaptic neurons may lead to strengthening of the synapse between them. In various parts of the nervous system, correlated presynaptic and postsynaptic activity indeed results in long-term synaptic potentiation (LTP) (Bliss and Lømo, 1973; Bliss and Collingridge, 1993; Nicoll and Malenka, 1995; Katz and Shatz, 1996; Magee and Johnston, 1997; Markram et al., 1997; Malenka and Nicoll, 1999). Inherent in Hebb's postulate is the assumption of synapse specificity; only connections between neurons undergoing correlated activity become potentiated. Therefore individual synapses can be regarded as independent units for activityinduced synaptic modifications. This assumption has been incorporated into many neural network models (Churchland and Sejnowski, 1992; Rolls and Treves, 1998) and was consistent with some experimental studies on LTP (Kelso et al., 1986; Gustafsson et al., 1987; Brown et al., 1990; Malenka and Nicoll, 1999). However, there is growing evidence that synaptic modification induced by activity in one pathway may be accompanied by changes in the efficacy of other adjacent synapses (Lynch et al., 1977; Bonhoeffer et al., 1989, 1990; Kossel et al., 1990; Christie and Abraham, 1992; Schuman and Madison, 1994; Scanziani et al., 1996; Engert and Bonhoeffer, 1997). For example, LTP induced at synaptic inputs on a single CA1 pyramidal neuron in the hippocampus appears to spread to synapses formed by the same set of afferent fibers on the neighboring neurons (Bonhoeffer et al., 1989; Schuman and Madison, 1994). In cultured hippocampal slices, LTP induced at one set of synaptic inputs to CA1 pyrami-

\footnotetext{
Received Nov. 17, 1999; revised Jan. 12, 2000; accepted Feb. 7, 2000.

This work was supported by National Institutes of Health Grant NS 36999. G.-q.B. was supported by a University of California Presidential fellowship and National Institutes of Health Training Grant NS 07220. We thank X.-y. Wang for culture preparations and B. Berninger, Y. Goda, and D. Hagler for helpful discussions.

H.-z.W.T., L.I.Z., and G.-q.B. contributed equally to this work.

Correspondence should be addressed to Dr. Mu-ming Poo at the above address. E-mail: mpoo@biomail.ucsd.edu.

Copyright (C) 2000 Society for Neuroscience $\quad 0270-6474 / 00 / 203233-11 \$ 15.00 / 0$
}

dal neurons also spreads to nearby synapses on the same postsynaptic neuron (Engert and Bonhoeffer, 1997), resulting in a "breakdown" of input specificity. In cultures of dissociated hippocampal neurons, induction of long-term depression (LTD) at glutamatergic synapses is accompanied by a backpropagation of depression to input synapses on the dendrites of the presynaptic neuron. The depression also spreads laterally to divergent outputs of the presynaptic neuron and to convergent inputs of the postsynaptic cell (Fitzsimonds et al., 1997).

In the present study, we used cultures of dissociated hippocampal neurons to characterize the spread of LTP in defined neural networks. Using perforated whole-cell patch clamp, we simultaneously monitored all synaptic connections within the networks of three or four neurons. After the induction of LTP at one excitatory connection by correlated presynaptic and postsynaptic excitation, we found significant potentiation at other connections that did not experience correlated activity. Potentiation was found only in a subset of synaptic connections that are directly associated with the presynaptic neuron involved in the induction of LTP, including synapses made onto its dendrites and synapses made by its axon collaterals on glutamatergic neurons. These results imply the existence of a long-range cytoplasmic signaling within the presynaptic neuron and add a new dimension to activity-induced synaptic modification at the network level that bears direct implications to developmental remodeling and learning functions of the neural network.

\section{MATERIALS AND METHODS}

Cell culture. Low-density cultures of dissociated embryonic rat hippocampal neurons were prepared as described previously (Wilcox et al., 1994). Hippocampi were removed from embryonic day 18 (E18) to E20 embryonic rats and treated with trypsin for $20 \mathrm{~min}$ at $37^{\circ} \mathrm{C}$, followed by washing and gentle trituration. The dissociated cells were plated on poly-L-lysine-coated glass coverslips in $35 \mathrm{~mm}$ Petri dishes with 30,00090,000 cells per dish. The culture medium was DMEM (BioWhittaker, Walkersville, MD) supplemented with $10 \%$ heat-inactivated fetal bovine serum (HyClone, Logan, UT), 10\% Ham's F12 with glutamine (BioWhittaker), and $50 \mathrm{U} / \mathrm{ml}$ penicillin-streptomycin (Sigma, St. Louis, 
MO). Twenty-four hours after plating, one-third of the culture medium was replaced by the same medium supplemented with $20 \mathrm{~mm} \mathrm{KCl}$. Both glial cells and neuronal cell types are present under these culture conditions. To avoid a complication of connectivity with other neurons not monitored by our recording, we have chosen to examine only triplets or quadruplets found on isolated patches of glial cells. In cases in which connections with a few other nearby unmonitored neurons were suspected, we have used a suction pipette to remove physically these nearby cells.

Electrophysiology. Simultaneous whole-cell perforated-patch recordings (Hamill et al., 1981; Rae et al., 1991) from three or four hippocampal neurons were performed with patch-clamp amplifiers (Axopatch 200; Axon Instruments) at room temperature $\left(22-25^{\circ} \mathrm{C}\right)$. The internal solution contained the following (in $\mathrm{mM}$ ): potassium gluconate 136.5, $\mathrm{KCl} 17.5$, $\mathrm{NaCl} 9, \mathrm{MgCl}_{2} 1$, HEPES 10, EGTA 0.2 , and $200 \mu \mathrm{g} / \mathrm{ml}$ amphotericin B, $\mathrm{pH}$ 7.3. The external bath solution was HEPES-buffered saline containing the following (in mM): $\mathrm{NaCl} 150, \mathrm{KCl} 3, \mathrm{CaCl}_{2} 3, \mathrm{MgCl}_{2} 2$, HEPES 10 , and glucose $5, \mathrm{pH} 7.3$. The culture was constantly perfused with fresh bath medium at a rate of $0.5-1 \mathrm{ml} / \mathrm{min}$ throughout the recording. The neurons were visualized with a phase-contrast inverted microscope (Zeiss IM35). Signals (filtered at $5 \mathrm{kHz}$ ) were acquired at a sampling rate of $10 \mathrm{kHz}$ and analyzed with Axoscope software (Axon Instruments). Series resistance (15-35 M 2 ) was compensated at $80 \%$ (lag, $100 \mu \mathrm{sec}$ ). In general there is no change in series resistance and input impedance (200-500 M $\Omega$ ) after the repetitive-pairing protocol. Data were accepted for analysis only in the cases in which series resistance and input impedance did not vary $>10 \%$ throughout the experiment and the coefficient of variation of postsynaptic currents (PSCs) during the control period did not exceed 0.5 .

Mapping of network connectivity. For assaying synaptic connectivity, each neuron was stimulated at a low frequency $(0.025-0.05 \mathrm{~Hz})$ by $1 \mathrm{msec}$ step depolarization $(+100 \mathrm{mV})$ in the voltage-clamp mode $\left(\mathrm{V}_{c}=-70\right.$ $\mathrm{mV}$ ), and responses from all the other neurons as well as the autaptic response in the stimulated neuron itself were recorded simultaneously. The connection from one neuron to another is determined by the consistent appearance of monosynaptic EPSCs or IPSCs elicited by presynaptic stimulation with an onset latency of $\leq 4 \mathrm{msec}$ (variation $<1$ msec). To avoid ambiguity in the interpretation of our results, we have examined only those circuits in which recordings of PSCs showed no polysynaptic responses with long onset latencies. The GABAergic connections are usually identified by the slow time course and the negative reversal potential $(-60$ to $-40 \mathrm{mV}$ ) of the postsynaptic current. Pharmacological studies using selective receptor antagonists have further confirmed that EPSCs are mediated by AMPA receptors and IPSCs are mediated by $\mathrm{GABA}_{\mathrm{A}}$ receptors (Fitzsimonds et al., 1997; Bi and Poo, 1998).

\section{RESULTS}

\section{Networks of cultured hippocampal neurons}

Cultures of dissociated rat hippocampal neurons were prepared from E18 to E20 rat embryos and used after 8-14 d in vitro. To assess the connectivity and synaptic changes within a small network of three (triplets) or four (quadruplets) interconnected neurons, whole-cell perforated-patch recordings of synaptic currents were made simultaneously from the soma of all the cells in the network (Fig. 1A). For quadruplets, there are potentially 16 different connections among these neurons, with each connection consisting of tens to hundreds of synaptic contacts (boutons). The connectivity of the network was determined by recording evoked EPSCs or IPSCs simultaneously from all four neurons in response to sequential stimulation of each one of them (see Materials and Methods). A schematic drawing in Figure $1 B$ depicts the connectivity of the network, with each crossing point representing one synaptic connection formed between a pair of presynaptic and postsynaptic cells. Connections in the same row share a common presynaptic neuron, whereas those in the same column share a common postsynaptic cell.

In a typical experiment, LTP was induced at a glutamatergic connection (Bi and Poo, 1998) by repetitive correlated stimulation of the presynaptic neuron (voltage-clamped at $-80 \mathrm{mV}$ to prevent spiking resulting from reciprocal inputs) and postsynaptic cell (in current clamp to allow spiking) at $1 \mathrm{~Hz}$ for $80 \mathrm{sec}$ (Fig. $1 C)$. Each presynaptic stimulus (1 msec step depolarization of $+100 \mathrm{mV}$ ) was followed within $10 \mathrm{msec}$ by a postsynaptic spike, resulting from either suprathreshold excitation by the stimulated connection itself (Fig. 1C, top traces) or injection of a depolarizing current pulse ( $1 \mathrm{msec} ; 2 \mathrm{nA})$ when the stimulated connection was subthreshold (bottom traces). When applied to glutamatergic connections formed on GABAergic neurons, this correlated stimulation did not result in LTP (Bi and Poo, 1998). We thus focused our study only on the networks consisting of at least two glutamatergic neurons, and LTP was induced at a connection between them. Because repetitive stimulation of a neuron with an autaptic connection under current clamp could induce change of this connection ( $\mathrm{Bi}$ and $\mathrm{Poo}, 1998)$, we only chose glutamatergic neurons without autapses as postsynaptic neurons for the induction of LTP to avoid ambiguity in the interpretation.

For studying the spread of LTP within the network, LTP was induced at a connection between two excitatory neurons arbitrarily designated cells 2 and 1 for presynaptic and postsynaptic cells, respectively (Fig. $1 B$, see the short arrow), and changes in the synaptic strength of all other connections were monitored. These changes could reflect the possible spread of synaptic modification in different directions: (1) to synapses on the dendrites of neuron 2 (backpropagation), (2) to divergent outputs of neuron 2 (presynaptic lateral propagation), (3) to convergent inputs on neuron 1 (postsynaptic lateral propagation), and (4) to output synapses made by neuron 1 (forward propagation). The spread of synaptic changes can be conveniently depicted by a $4 \times 4$ matrix corresponding to the 16 connections within the quadruplet (Fig. $1 D)$, with each element of the matrix displaying the inferred direction of propagation when the corresponding connection undergoes changes. Synaptic changes at an autaptic connection (of cell 1 or 2) or the recurrent connection (from cell 1 to 2) can reflect two different forms of propagation. The spread of potentiation to connections that are only associated with neurons not involved in the induction of LTP is referred to as "secondary propagation."

\section{Propagation of LTP in networks of glutamatergic neurons}

We first examined the spread of LTP within small networks consisting only of glutamatergic neurons. In the examples shown in Figure 2, each network consisted of four glutamatergic neurons (referred to as $E_{1}$ to $E_{4}$ ) that were simultaneously recorded by perforated whole-cell patch clamp. Correlated stimulation was repetitively applied to neurons $\mathrm{E}_{2}$ and $\mathrm{E}_{1}$ to induce LTP at the connection made by $E_{2}$ on $E_{1}$ (referred to as $E_{2} \rightarrow E_{1}$ ) that was either subthreshold (Fig. 2A) or suprathreshold (Fig. 2B). Each element of the matrix (shown in Fig. 2, top left panels) displays two superimposed traces of averaged EPSCs with all the cells voltage-clamped at $-70 \mathrm{mV}$ at 5-10 min before and 20-30 min after the correlated stimulation. The matrix also describes the connectivity of the network, which is schematically shown by the drawing (Fig. 2, top right panels). During the repetitive stimulation, $\mathrm{E}_{1}$ was kept in current clamp to allow spiking, while all other neurons including $\mathrm{E}_{2}$ were voltage-clamped at $-80 \mathrm{mV}$. The amplitudes of EPSCs of various connections during the course of the experiment were sampled at $0.025 \mathrm{~Hz}$ (Fig. 2, bottom panels). In the first example (Fig. $2 A$ ), correlated stimulation induced LTP at connection $\mathrm{E}_{2} \rightarrow \mathrm{E}_{1}$. Besides $\mathrm{E}_{2} \rightarrow \mathrm{E}_{1}$, significant increases 
A

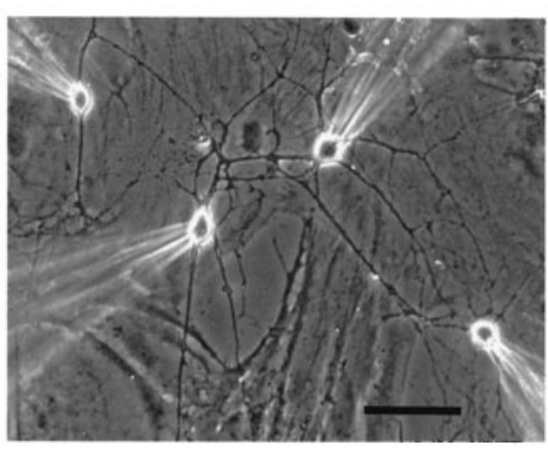

B

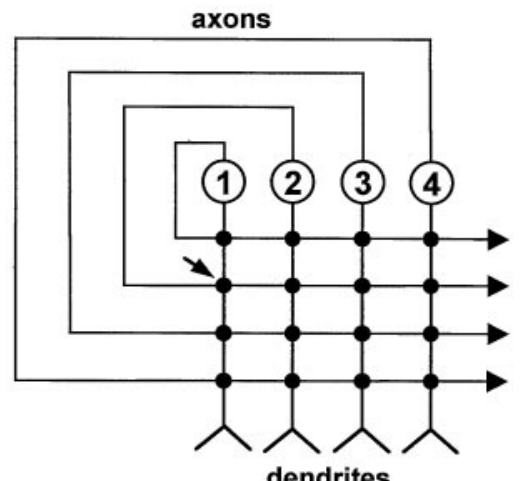

\section{C}
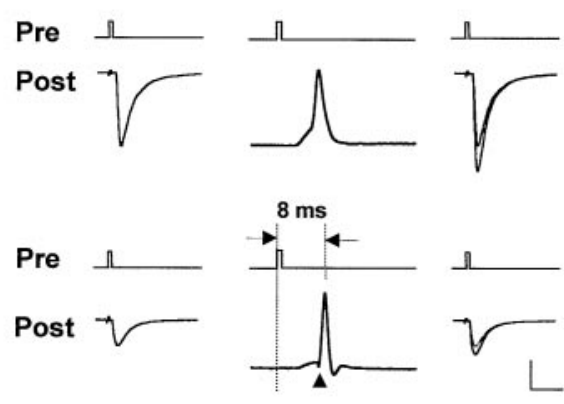

before

induction

after

induction

D

\begin{tabular}{|c|c|c|c|c|}
\hline $\begin{array}{c}\text { post } \\
\text { pre }\end{array}$ & 1 & 2 & 3 & 4 \\
\hline 1 & $\begin{array}{c}\text { forward } \\
\text { post-lat }\end{array}$ & $\begin{array}{c}\text { forward } \\
\text { back }\end{array}$ & forward & forward \\
\hline 2 & induction & $\begin{array}{c}\text { pre-lat } \\
\text { back }\end{array}$ & pre-lat & pre-lat \\
\hline 3 & post-lat & back & second & second \\
\hline 4 & post-lat & back & second & second \\
\hline
\end{tabular}

Figure 1. Small neural networks of cultured hippocampal neurons. A, A microscopic image of a network of four interconnected neurons (quadruplet) in a $14 \mathrm{~d}$ hippocampal culture, together with whole-cell recording electrodes. Scale bar, $50 \mu \mathrm{m}$. B, A schematic drawing depicting all possible synaptic connections among four neurons. The short arrow indicates the connection (made by neuron 2 on neuron 1 ) chosen for the induction of LTP. $C$, Two examples of recordings illustrating two different protocols for the induction of LTP. The left and right traces represent sample traces of EPSCs (average of 20 consecutive traces) at 5-10 min before and 20-30 min after the induction of LTP, respectively, with the cells voltage-clamped at $-70 \mathrm{mV}$. The middle traces (average of 5) depict postsynaptic potentials during repetitive presynaptic stimulation ( $1 \mathrm{msec}$ step depolarization of $+100 \mathrm{mV}$ at $1 \mathrm{~Hz}$ for $80 \mathrm{sec}$ ). In the first protocol (top traces), used for the suprathreshold connections, postsynaptic spiking was elicited directly by the presynaptic stimulation. In the second protocol (bottom traces), used for the subthreshold connections, depolarizing current pulses (1 msec; $2 \mathrm{nA}$ ) were injected into the postsynaptic neuron to trigger a spike that peaked within $10 \mathrm{msec}$ after the presynaptic stimulation. The arrowhead marks the onset of the injected current pulse. Calibration: $200 \mathrm{pA}, 15 \mathrm{msec}$ (left, right); $40 \mathrm{mV}, 6 \mathrm{msec}$ (middle). D, A matrix indicating potential forms of the spread of LTP, with each element displaying the inferred direction of propagation when the connection corresponding to that shown in $B$ undergoes synaptic potentiation. back, backpropagation; forward, forward propagation; pre-lat, presynaptic lateral propagation; post-lat, postsynaptic lateral propagation; second, secondary propagation.

in EPSC amplitude were also observed at several other connections (data plotted in red). These included connections $\mathrm{E}_{2} \rightarrow \mathrm{E}_{4}$ and $E_{3} \rightarrow E_{2}$, suggesting presynaptic lateral propagation and backpropagation, respectively. Neither forward propagation to $\mathrm{E}_{1} \rightarrow \mathrm{E}_{3}$ nor postsynaptic lateral propagation to $\mathrm{E}_{3} \rightarrow \mathrm{E}_{1}$ was found. However, the recurrent connection $\mathrm{E}_{1} \rightarrow \mathrm{E}_{2}$ became potentiated, consistent with the backpropagation of LTP. Finally, there was no secondary propagation of potentiation to $E_{4} \rightarrow E_{3}, E_{4} \rightarrow E_{4}$, and $\mathrm{E}_{3} \rightarrow \mathrm{E}_{3}$. In the second example shown in Figure $2 B$, the spread of potentiation was similarly restricted to the synapses associated with $E_{2}$, including the autaptic connection $E_{2} \rightarrow E_{2}$, the potentiation of which can be attributed to either presynaptic lateral propagation or backpropagation.

In the above experiments, the recurrent connection $\mathrm{E}_{1} \rightarrow \mathrm{E}_{2}$ (Fig. $2 A$ ) and the autaptic connection $\mathrm{E}_{2} \rightarrow \mathrm{E}_{2}$ (Fig. $2 B$ ) became potentiated. This result may seem puzzling based on the previous finding of the importance of spike timing in determining the direction of synaptic modification. At these synapses, the evoked synaptic input immediately followed the brief step depolarization of $\mathrm{E}_{2}$, which appears to be similar to "negatively correlated spiking" that has been shown to induce LTD (Markram et al., 1997; Bi and Poo, 1998; Zhang et al., 1998). However, there is a crucial difference between the current and previously used conditions. For negatively correlated spiking, the postsynaptic cell is under current clamp to allow depolarization by synaptic input and firing of the action potential. In the present study, the "postsynaptic" cell $\mathrm{E}_{2}$ was under voltage clamp, and the stimulus was a brief step depolarization. Furthermore, it has been shown that depolarization by EPSP may result in significant $\mathrm{Ca}^{2+}$ influx through L-type channels (Mermelstein et al., 2000), which is essential for the induction of LTD (Bi and Poo, 1998). With the postsynaptic membrane potential clamped, the brief step depolarization coupled with synaptic current may not open a sufficient number of L-type channels and thus may be unable to induce LTD. On the other hand, the LTP that resulted at $E_{1} \rightarrow E_{2}$ is consistent with backpropagation, whereas LTP at the autaptic 
A
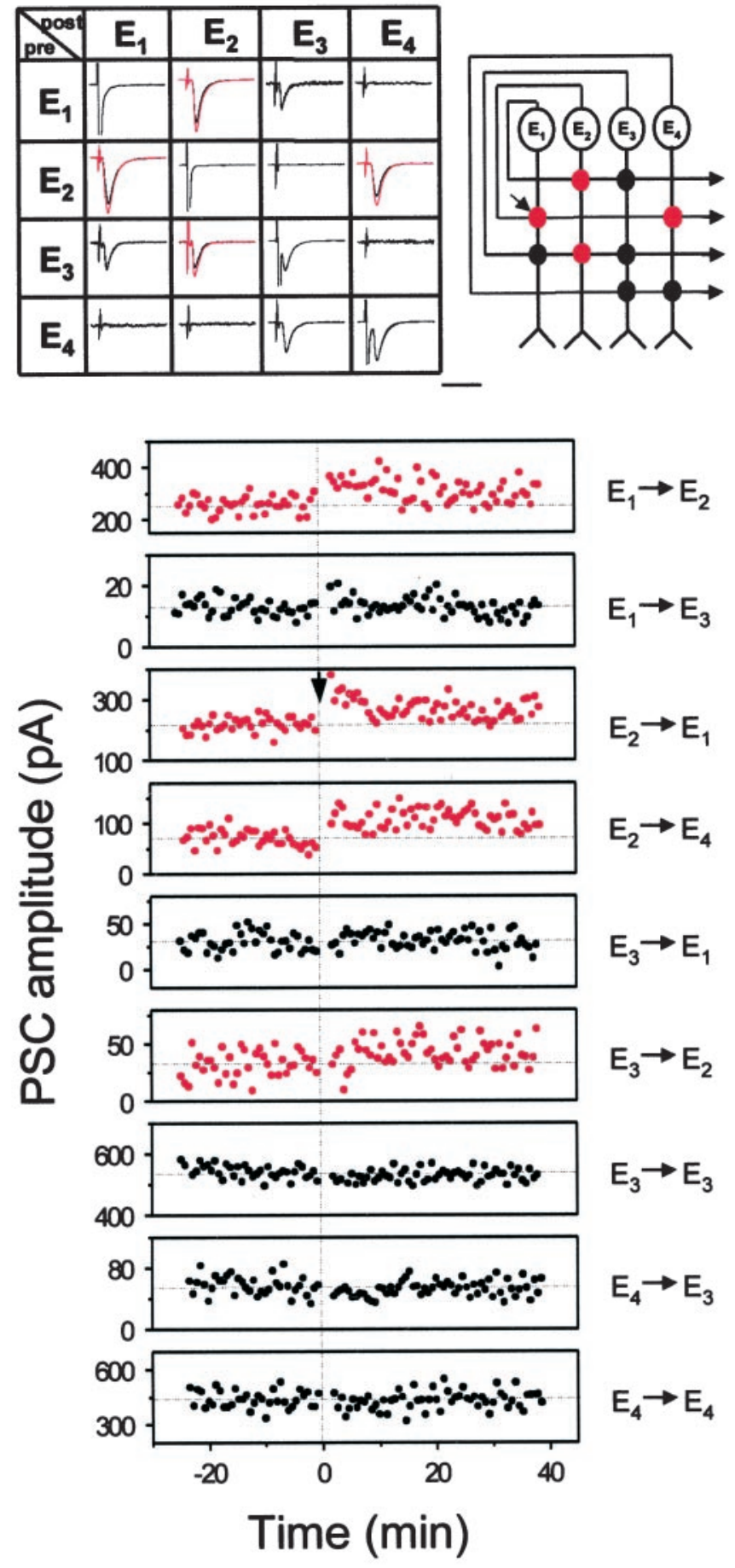

B
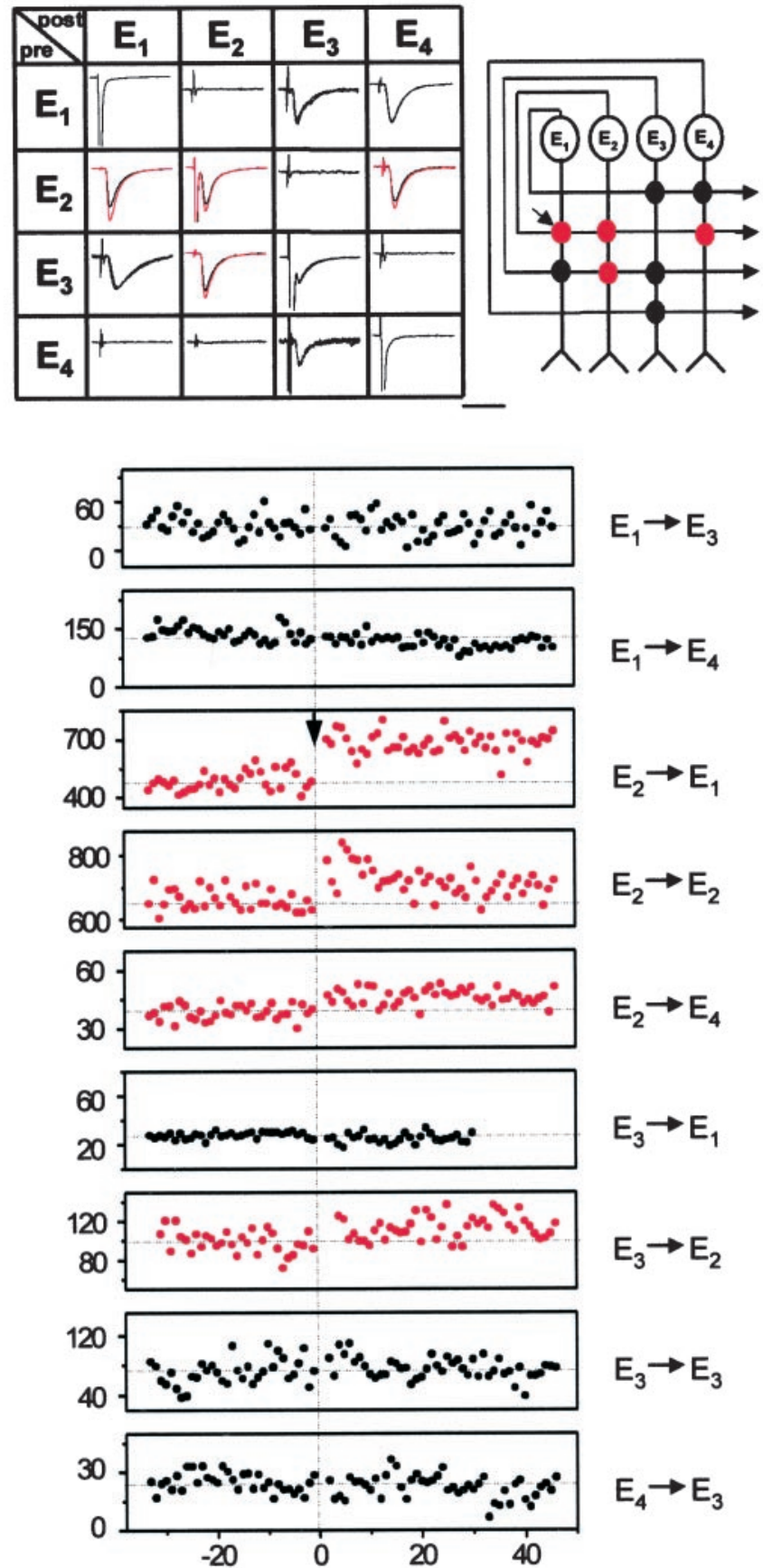

Time (min)

Figure 2. Propagation of LTP in the networks of glutamatergic neurons. $A$, An example of results obtained from a quadruplet that consisted of only glutamatergic neurons (referred to as $E_{i}$ ) is shown. Top Left, The matrix depicts PSCs observed for all possible monosynaptic connections within the quadruplet. pre and post indicate the presynaptic and postsynaptic cell, respectively, for the connection corresponding to each matrix element. For each connection, two superimposed traces of PSCs (each represents the average of 20 consecutive traces) are shown for recordings made at 5-10 min before and 20-30 min after the induction of LTP (at $\mathrm{E}_{2} \rightarrow \mathrm{E}_{1}$ ) with all the cells held at $-70 \mathrm{mV}$. Red traces were used to mark those connections in which the PSC amplitude showed a significant increase $(>15 \%)$ after LTP induction. In all other connections, two traces showed nearly complete overlap. Autaptic currents were preceded by a large stimulation artifact, which is observed in all diagonal elements $\mathrm{E}_{i} \rightarrow \mathrm{E}_{i}$. Calibration: 25 msec (also B). Top Right, The schematic drawing depicts the connectivity within the quadruplet, with dots representing the functional connections and red dots indicating the connections that exhibited potentiation after the induction of LTP at $\mathrm{E}_{2} \rightarrow \mathrm{E}_{1}$. Bottom, The amplitudes of PSCs observed over the course of the experiment for all connections with detectable synaptic transmission were plotted. The short arrow marks the time of LTP induction (at $\left.\mathrm{E}_{2} \rightarrow \mathrm{E}_{1}\right)$. Data for connections that showed significant potentiation after LTP induction are plotted in red. $B$, Data from another quadruplet similar to that shown in $A$ are presented. 


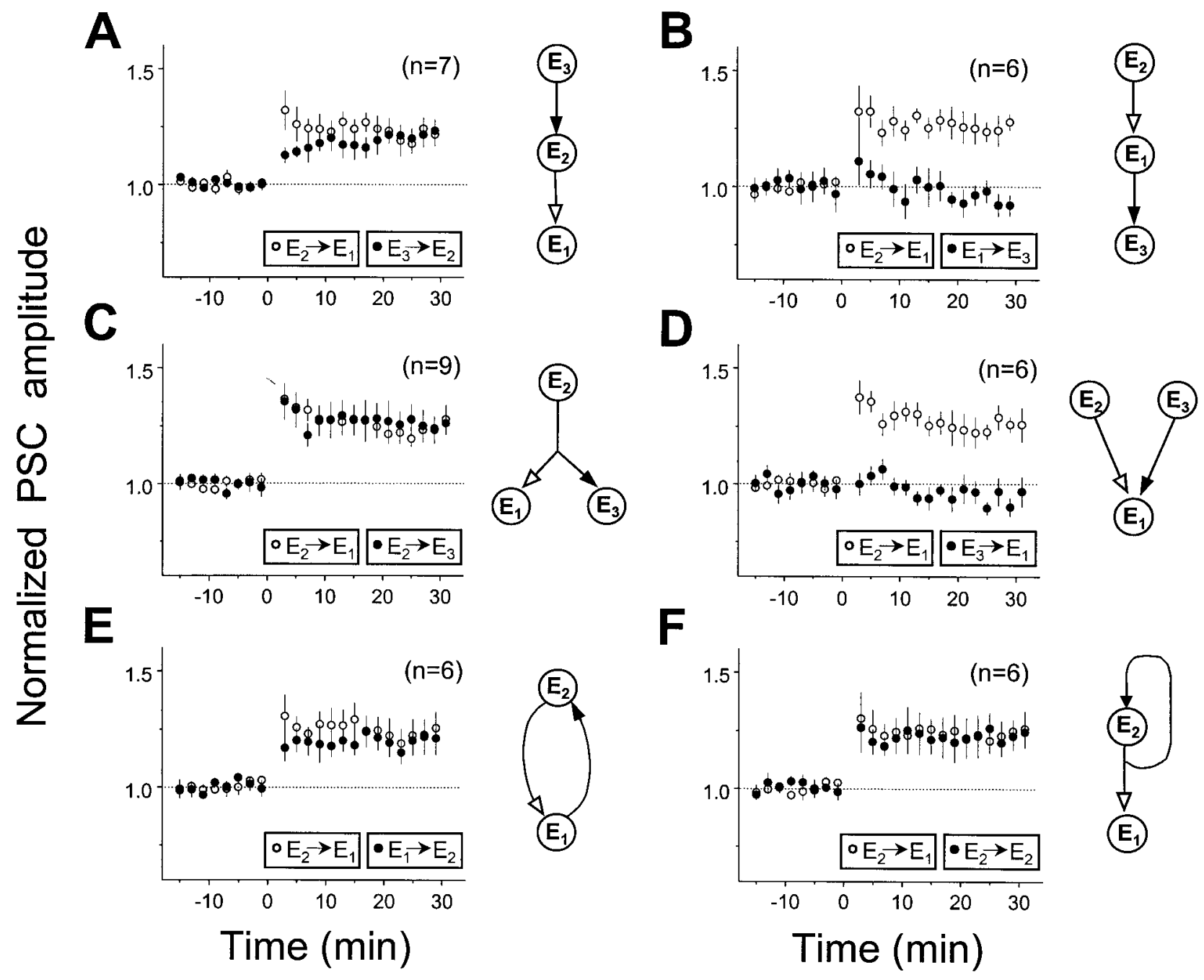

Figure 3. Summary of propagation of LTP in networks of glutamatergic neurons. Results were subdivided according to the circuit configurations, depicted schematically in the drawings on the right, as serial $(A, B)$, divergent $(C)$, convergent $(D)$, recurrent $(E)$, and autaptic $(F)$ connections. Arrows represent glutamatergic connections. The site of LTP induction is designated $\mathrm{E}_{2} \rightarrow \mathrm{E}_{1}$ and marked by the open arrow. Data for $\mathrm{E}_{2} \rightarrow \mathrm{E}_{1}$ are plotted in open circles, and those for the connections under examination for propagation are plotted in filled circles. Data points (mean \pm SEM) represent the averaged amplitude of PSCs. The amplitude of PSCs from each experiment was grouped with a $2 \mathrm{~min}$ bin and normalized against the mean value observed before the repetitive stimulation. $n$ refers to the number of specific connections in which data were collected for the corresponding configuration. All data were obtained from 5 quadruplets and 11 triplets. Significant potentiation of the examined connection at 20-30 min after the induction of LTP was found for configurations shown in $A, C, E$, and $F$ ( $p<0.01$, Student's $t$ test). In these experiments, six of seven cases in $A$, seven of nine cases in $C$, four of six cases in $E$, and five of six cases in $F$ showed significant potentiation (with a $>15 \%$ increase of synaptic strength) at the propagated site.

connection $\mathrm{E}_{2} \rightarrow \mathrm{E}_{2}$ is consistent with presynaptic lateral propagation and backpropagation.

To analyze quantitatively the spread of potentiation, we subdivided data obtained from networks of three or four neurons into subcategories of serial, divergent, convergent, recurrent, and autaptic connections relative to the site of induction of LTP $\left(E_{2} \rightarrow E_{1}\right)$. The amplitudes of EPSCs of specific connections after the induction of glutamatergic LTP at $E_{2} \rightarrow E_{1}$ were normalized and averaged for data collected from all experiments. To avoid ambiguity in inferring the direction of propagation, data that could be assigned to one and only one subcategory were included in this analysis. As shown in the results summarized from 5 quadruplets and 11 triplets (Fig. 3), the observations described above for the specific examples shown in Figure 2 were confirmed. There was backpropagation (Fig. $3 A$ ) and presynaptic lateral propagation (Fig. $3 C$ ) but no forward propagation (Fig. $3 B$ ) or postsynaptic lateral propagation (Fig. $3 D$ ). Potentiation of the recurrent connection (Fig. $3 E$ ) can be attributed to backpropagation, whereas that of the autaptic connection of $\mathrm{E}_{2}$ (Fig. $3 F$ ) can be attributed to presynaptic lateral propagation or backpropagation.

\section{Propagation of LTP in networks containing GABAergic neurons}

In two other examples shown in Figure 4, the quadruplet or triplet included a GABAergic neuron (indicated by I). In the case shown in Figure $4 A$, after the induction of LTP at $\mathrm{E}_{2} \rightarrow \mathrm{E}_{1}$, we observed backpropagation $\left(\mathrm{E}_{1} \rightarrow \mathrm{E}_{2}, \mathrm{E}_{2} \rightarrow \mathrm{E}_{2}\right.$, and $\mathrm{I}_{4} \rightarrow \mathrm{E}_{2}$ ) as well as lateral propagation $\left(E_{2} \rightarrow E_{3}\right.$ and $\left.E_{2} \rightarrow E_{2}\right)$ of potentiation. All other connections remained unchanged, including that made by the presynaptic neuron onto the GABAergic neuron $\left(E_{2} \rightarrow I_{4}\right)$. Similar presynaptic spread of potentiation was found in the case shown in Figure $4 B$. In both cases, backpropagation of potentiation to both glutamatergic and GABAergic neurons was ob- 
A
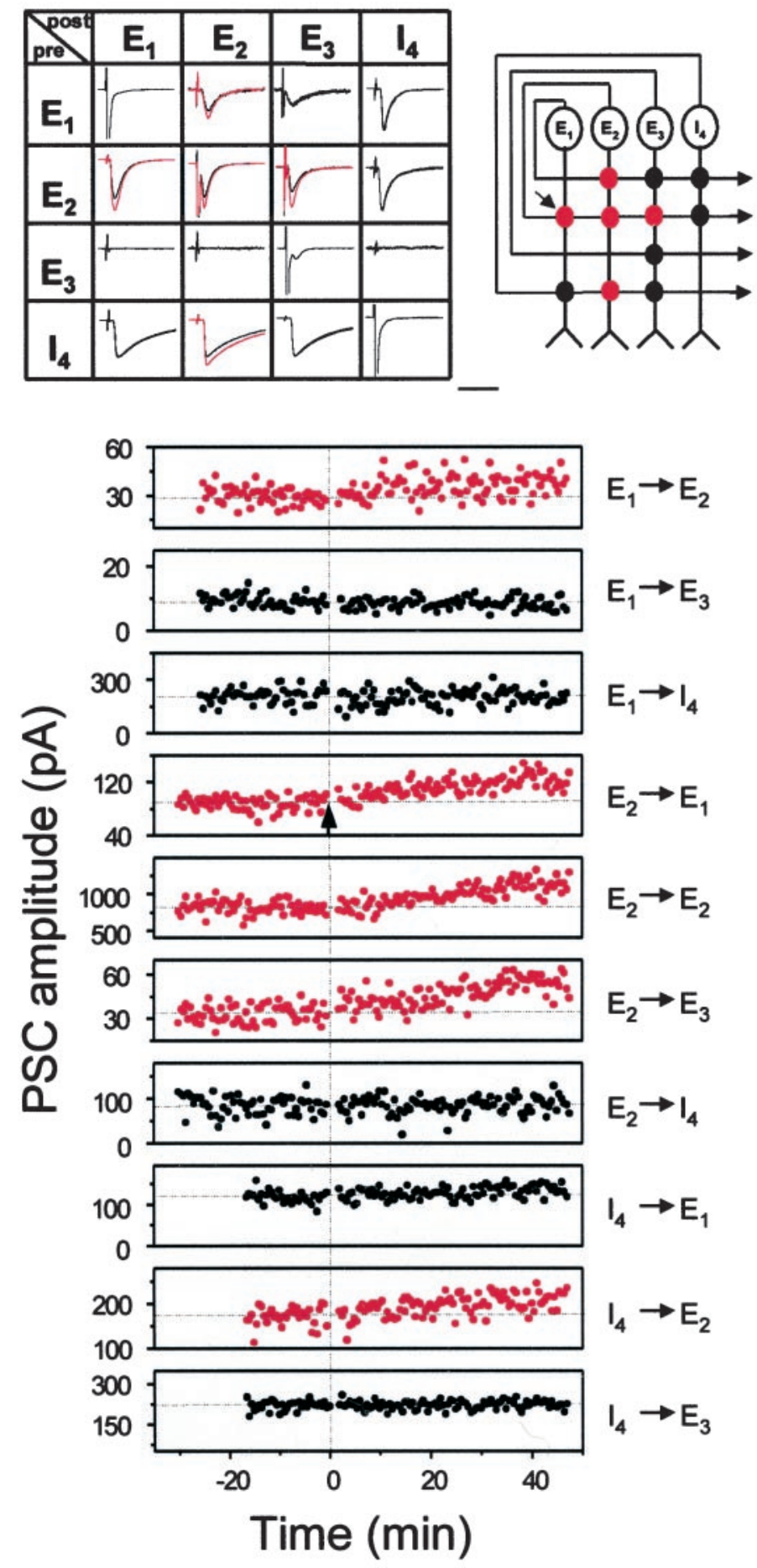
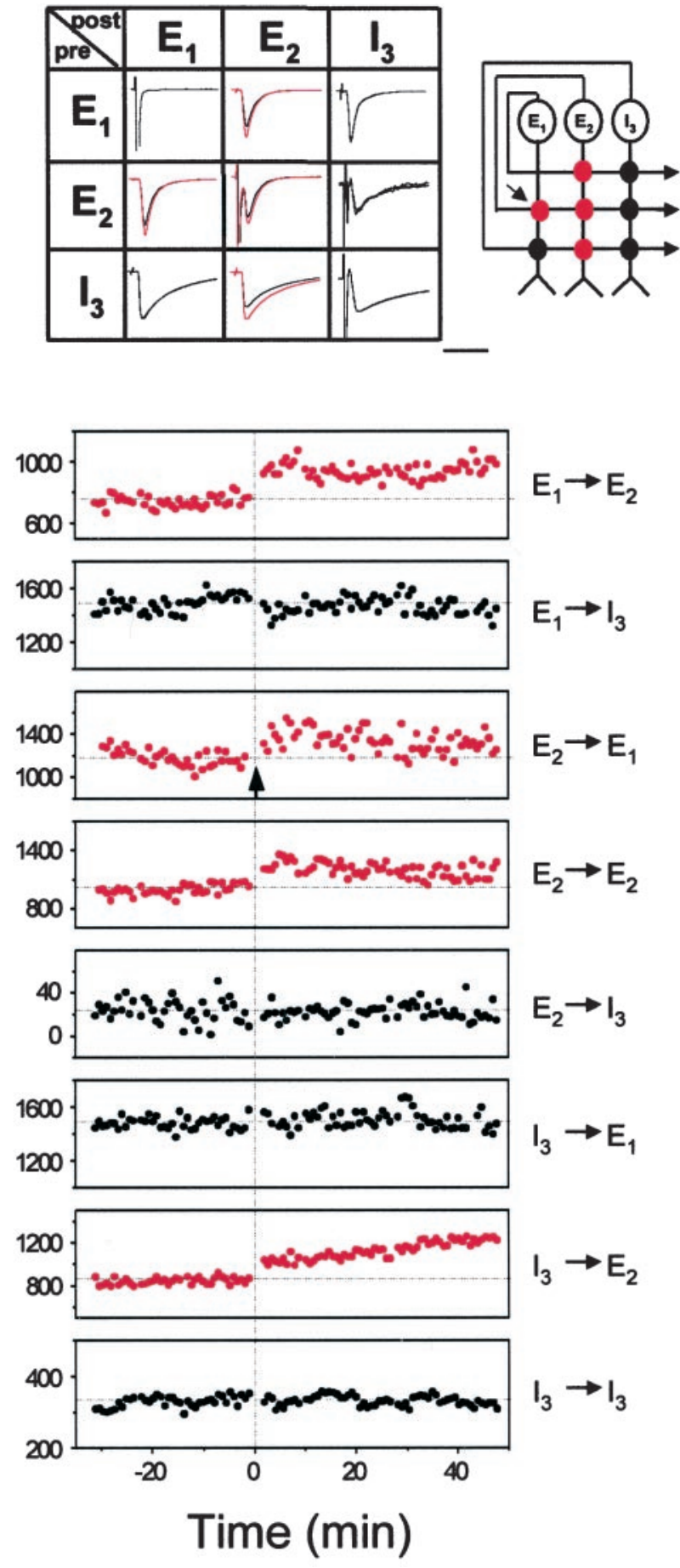

Figure 4. Propagation of LTP in networks containing GABAergic neurons. $A$, A quadruplet example of experiments that included one GABAergic neuron (designated $I_{i}$ ). $B$, A triplet example containing a GABAergic neuron. Both cases are presented in the same manner described in Figure 2.

served, but lateral propagation occurred only at connections made onto glutamatergic neurons. Therefore lateral propagation of LTP is target specific, reminiscent of target cell-dependent synaptic properties reported in other studies of synaptic plasticity (Maccaferri and McBain, 1996; McMahon and Kauer, 1997; Bi and Poo, 1998; Maccaferri et al., 1998; Reyes et al., 1998).
Experiments involving GABAergic neurons are summarized in Figure 5, in which data were subcategorized into four different groups according to cell types and connectivity configurations. There was backpropagation of LTP to GABAergic inputs on the presynaptic neuron (Fig. $5 A$ ) but no forward or postsynaptic lateral propagation (Fig. $5 B, D$ ). In contrast to the case for syn- 


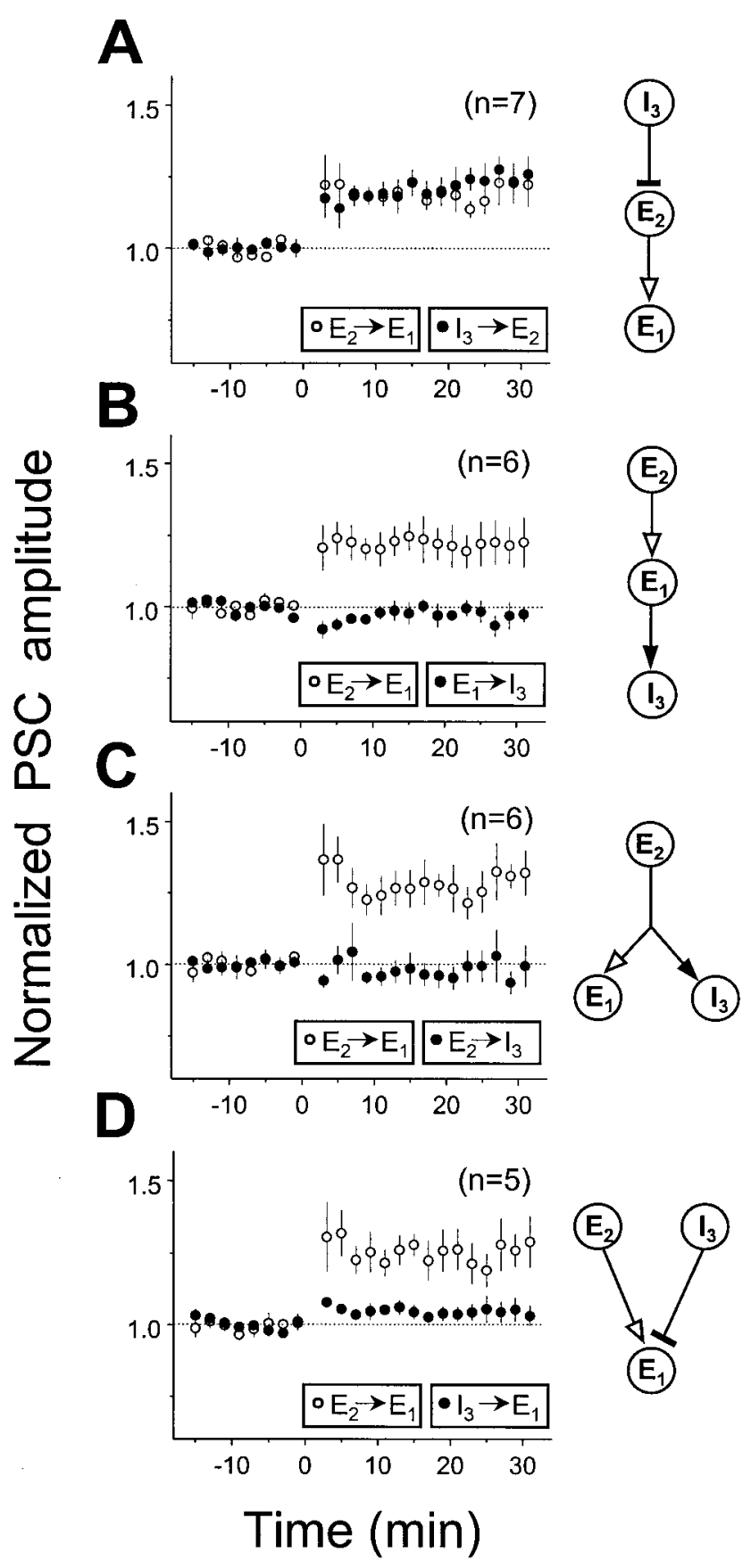

Figure 5. Summary of propagation of LTP in networks containing GABAergic neurons. Results are subdivided according to the corresponding circuit configurations $(A-D)$, and data are plotted as described in Figure 3. The bars indicate GABAergic synapses. All data were obtained from three quadruplets and seven triplets (including some cases that were used for data shown in Fig. 3). Significant potentiation of the examined connection at 20-30 min after the induction of LTP was found for the configuration shown in $A$ ( $p<0.01$, Student's $t$ test; 6 of 7 cases showed potentiation at the propagated site).

apses made onto glutamatergic neurons (Fig. 3C), presynaptic lateral propagation did not occur at synapses made onto GABAergic neurons (Fig. 5C). Thus the synapse specificity of LTP for inputs to the postsynaptic cell is preserved in the present hippocampal culture system, consistent with previous findings on developing Xenopus retinotectal connections (Zhang et al., 1998).

\section{Propagated potentiation requires induction of LTP}

In the above experiments, propagated potentiation to synapses associated with the presynaptic neuron $\mathrm{E}_{2}$ cannot be accounted for simply by the presence of repetitive depolarizations of $E_{2}$ during the induction of LTP at $\mathrm{E}_{2} \rightarrow \mathrm{E}_{1}$. This is suggested by the observation that there was no significant change in the EPSC amplitude of connections made by $E_{1}\left(E_{1} \rightarrow E_{3}\right.$ and $\left.E_{1} \rightarrow E_{4}\right)$ or received by $\mathrm{E}_{1}\left(\mathrm{E}_{3} \rightarrow \mathrm{E}_{1}, \mathrm{I}_{3} \rightarrow \mathrm{E}_{1}\right.$, and $\left.\mathrm{I}_{4} \rightarrow \mathrm{E}_{1}\right)$ (Figs. $3 B, D, 5 D$ ), which had also experienced the same number of repetitive depolarizations during the induction period. Further experiments were performed to examine directly the effects of repetitive presynaptic depolarization by voltage-clamping all neurons during the repetitive stimulation. An example of the result is shown in Figure $6 A$. In this triplet consisting of two glutamatergic and one GABAergic neuron, all three cells were held under voltage clamp $(-80 \mathrm{mV})$ during the application of the repetitive stimulation ( $1 \mathrm{msec}$ step depolarization of $+100 \mathrm{mV}$ at $1 \mathrm{~Hz}$ for $80 \mathrm{sec}$ ) to $E_{2}$. After the repetitive stimulation, there was no LTP induced at $E_{2} \rightarrow E_{1}$, nor were there any changes in synaptic efficacy at all other functional connections within the triplet. A summary of all such voltage-clamping experiments is shown in Figure $6 B$. For comparison, data on the induction of LTP in the presence of postsynaptic spiking were also included. It is clear that LTP cannot be induced by repetitively depolarizing the presynaptic neuron alone, in the absence of postsynaptic spiking, and that no potentiation was observed at synapses made or received by the presynaptic neuron, including its autaptic connections. These results are consistent with the previous findings that repetitive correlated excitation of both presynaptic and postsynaptic neurons is required for the induction of LTP using the present paradigm of low-frequency stimulation (Bi and Poo, 1998; Zhang et al., 1998).

The requirement of induction of LTP for the propagated potentiation is further examined by the experiments in which the NMDA subtype of glutamate receptors was blocked by perfusing the culture with $50 \mu \mathrm{M} \mathrm{D}(-)$-2-amino-5-phosphonopentanoic acid (D-APV), a specific blocker of NMDA receptors. As shown in the example in Figure $6 C$, LTP failed to be induced at $\mathrm{E}_{2} \rightarrow \mathrm{E}_{1}$, and no propagated potentiation was observed at other connections. The results from nine triplets are shown in Figure $6 D$. Taken together, these results of voltage-clamping and APV experiments strongly support the idea that the propagated potentiation is causally related to the induction of LTP.

\section{No "secondary" upstream or downstream propagation}

We further examined quantitatively whether potentiation could propagate to more upstream or downstream synapses associated with neurons that were not involved in the induction of LTP. A thorough study of such secondary propagation (Fig. 1D) was feasible with the use of quadruplets. The results are summarized in Figure 7. Data from quadruplets and triplets with cell 3 having an autaptic connection were categorized according to three different configurations for secondary propagation beyond neurons $E_{1}$ and $E_{2}$, which were involved in the induction of LTP. For backpropagation, we examined secondary backpropagation or secondary presynaptic lateral propagation through either a glutamatergic (Fig. $7 A$ ) or a GABAergic (Fig. $7 B$ ) neuron. For presynaptic lateral propagation, we examined secondary forward or postsynaptic lateral propagation (Fig. $7 C$ ). As shown by the averaged changes in PSC amplitudes at 20-30 min after the induction of LTP, we observed significant propagation of poten- 
A
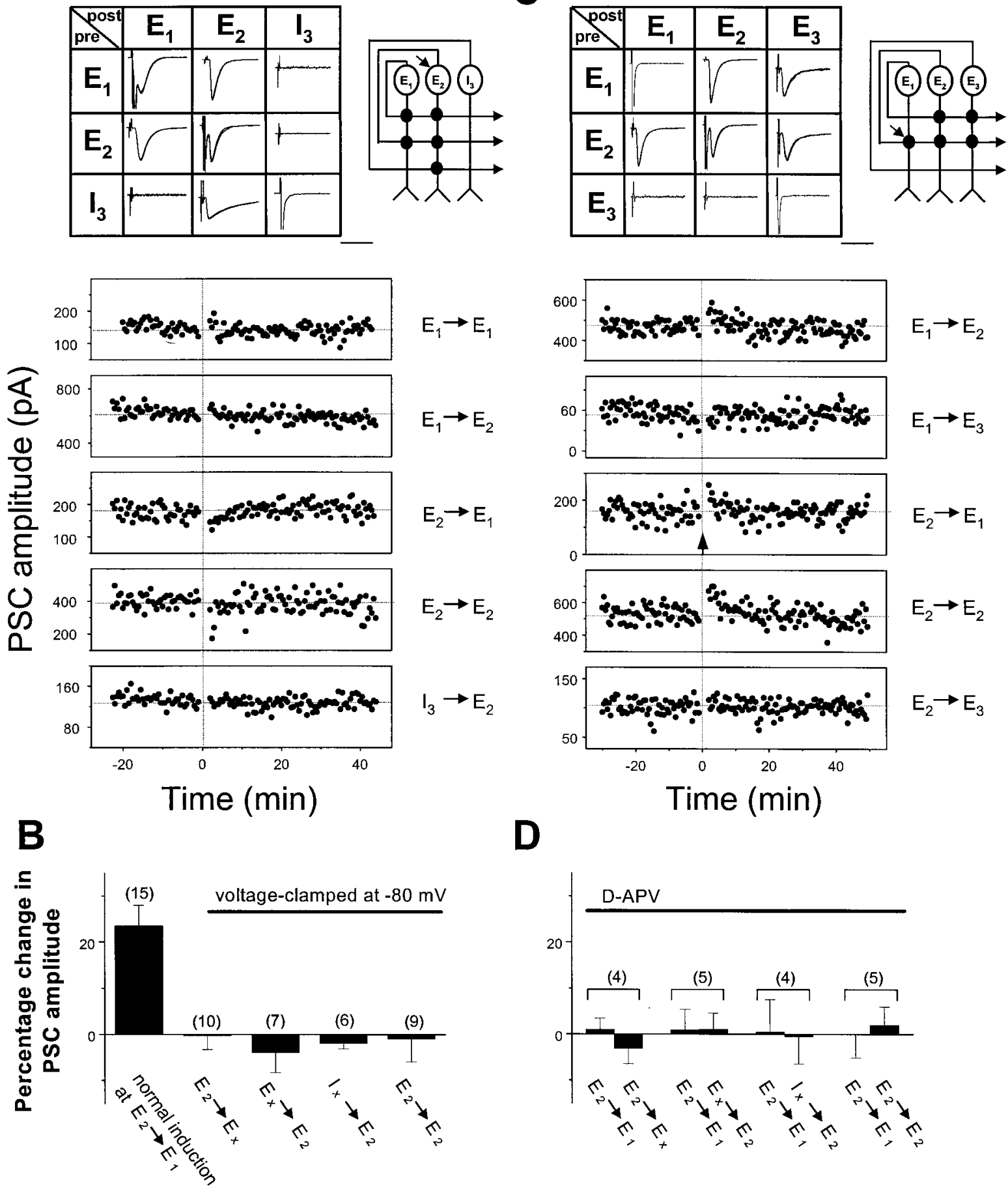

D

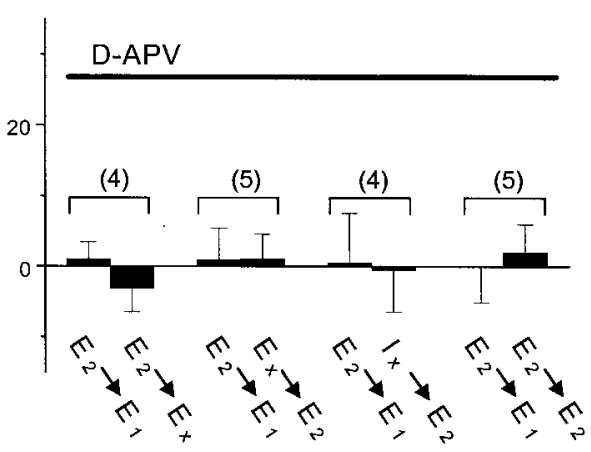

Figure 6. Propagated potentiation requires the induction of LTP. $A$, An example of voltage-clamping control experiments, using a triplet with two excitatory neurons and one GABAergic neuron. The experimental condition was similar to that shown in Figure $4 B$, except that all three cells were voltage-clamped at $-80 \mathrm{mV}$ during repetitive stimulation of $\mathrm{E}_{2}$ alone (marked by the short arrow). Data were shown in the same manner described in Figure 2. Note the absence of any persistent change of synaptic efficacy at all connections after repetitive stimulation of $\mathrm{E}_{2}$. $B$, Summary of experiments in which all neurons in the network were voltage-clamped at $-80 \mathrm{mV}$. The changes at the inputs, outputs, or autaptic connections of the presynaptic neuron $\mathrm{E}_{2}$ after repetitive stimulation were examined. The percentage change in the amplitude of EPSCs or IPSCs at each individual connection was calculated from the mean PSC amplitude at 20-30 min after the repetitive stimulation, as compared with the mean PSC amplitude observed during the control period. Vertical bars indicate the averaged percentage changes of PSC amplitude (mean \pm SD) for all connections examined (the total number shown by the number in parentheses associated with each bar). For comparison, the potentiation observed for cases in which the postsynaptic cell was held in current clamp is also shown, using the data set shown in Figure 3. $\mathrm{E}_{x}$ refers to excitatory neurons other than $\mathrm{E}_{2}$. $C$, An example experiment in which D-APV $(50 \mu \mathrm{M})$ was applied throughout the course of the experiment. There was no change of the PSC amplitude at any connection after repetitive correlated stimulation of $\mathrm{E}_{2} \rightarrow \mathrm{E}_{1}$. That cell 3 was glutamatergic was determined by its output to cell 4 (which is not shown). $D$, Summary of all experiments performed in the presence of D-APV. The averaged percentage change of PSC amplitude at the propagation sites (labeled below each vertical bar) after the induction protocol is plotted together with changes at the induction site $\left(\mathrm{E}_{2} \rightarrow \mathrm{E}_{1}\right)$ of the same set of networks. 


\section{Percentage change in PSC amplitude}

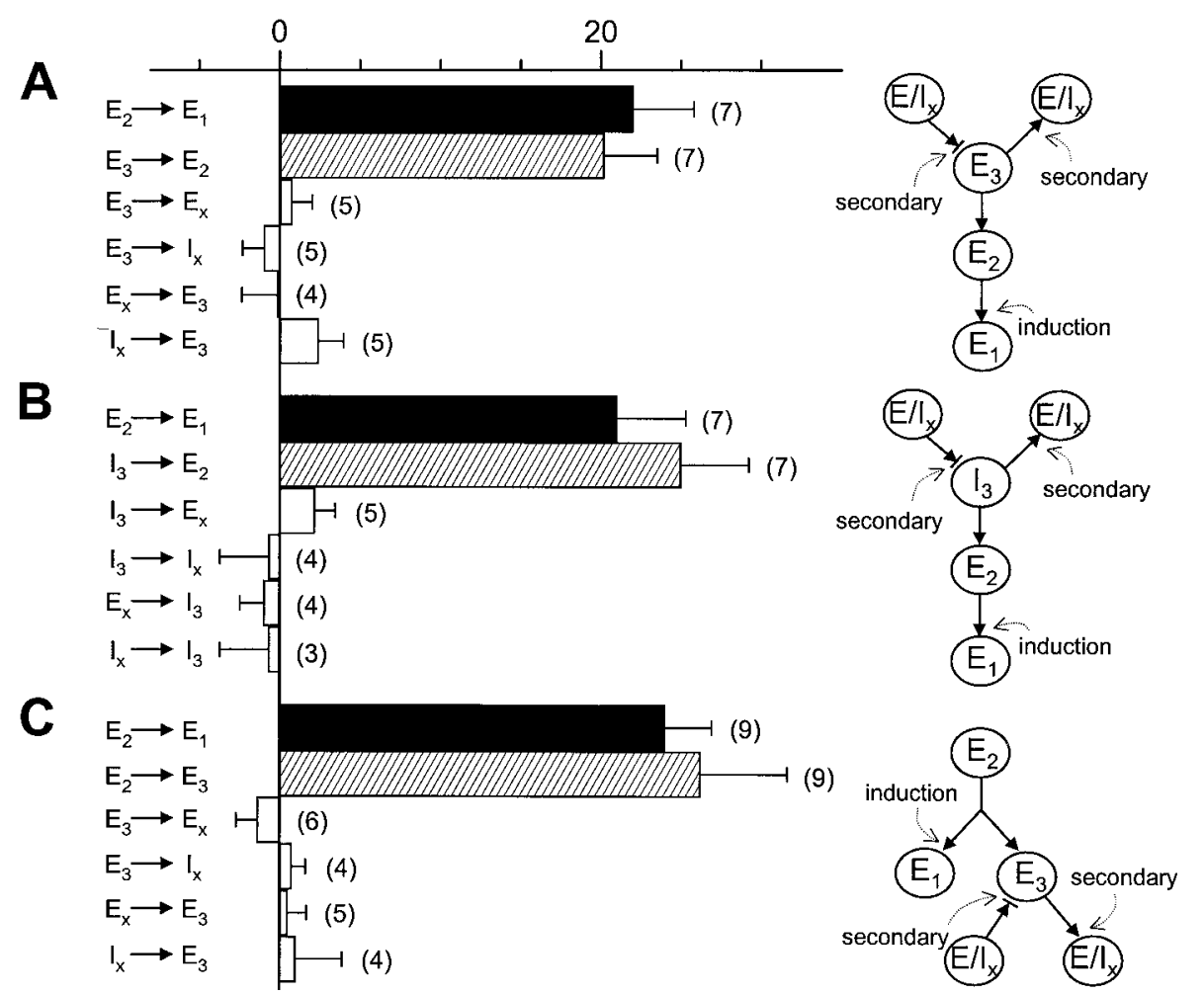

Figure 7. Summary of the secondary propagation of synaptic potentiation. $A-C$, Right, Schematic drawings depict the circuit configuration used in examining whether secondary propagation of LTP occurs. $\mathrm{E} / \mathrm{I}_{x}$ refers to neurons other than $\mathrm{E}_{2}$ or $\mathrm{E}_{1}$. Left, Bars indicate averaged percentage changes (mean $\pm \mathrm{SD}$; the total number examined shown by the number in parentheses to the right of each bar) in the amplitude of PSCs after LTP induction at $E_{2} \rightarrow E_{1}$. No significant potentiation was observed at any secondary connection, whereas a similar level of potentiation was found at connections associated with $\mathrm{E}_{2}$ and at the induction site $\left(\mathrm{E}_{2} \rightarrow \mathrm{E}_{1}\right)$. tiation to synapses associated with the presynaptic neuron $E_{2}$ but no secondary propagation in any cases.

\section{DISCUSSION}

Previous studies in hippocampal slices have shown that LTP can spread to nearby synapses. Such spread appears to be mediated by the action of an extracellularly diffusible factor(s) released from the activated synapse (Bonhoeffer et al., 1989; Kossel et al., 1990; Schuman and Madison, 1994; Engert and Bonhoeffer, 1997). In contrast, the spread of LTP we observed here is highly selectiveonly those synapses associated with the presynaptic neuron became potentiated. Using FM1-43 to stain a repetitively stimulated neuron (Betz and Bewick, 1992; Ryan et al., 1993), we found that synapses made by a single neuron are distributed rather haphazardly among other synapses in the network (data not shown). Physical proximity thus cannot account for the selectivity in the spread of potentiation. The simplest hypothesis to account for the selective spread is that a long-range cytoplasmic signaling within the presynaptic neuron is directly responsible for inducing potentiation at the propagated sites. However, we cannot exclude the possibility that an extracellularly diffusible factor could act selectively only on a set of synapses associated with the presynaptic neuron, if the induction of LTP had endowed the synapses associated with the presynaptic neuron a susceptibility to potentiation.

The induction of LTP in the present study requires postsynaptic spiking and activation of NMDA receptors (Bi and Poo, 1998; Zhang et al., 1998). The restricted presynaptic spread of potentiation thus suggests localized retrograde signaling at the site of induction of LTP, possibly via spatially restricted effects of a retrograde factor(s) or direct interactions between membranebound molecules (Williams et al., 1989; Schuman and Madison, 1994; Arancio et al., 1996; Wang and Poo, 1997). The cytoplasmic signal that propagates throughout the presynaptic neuron is likely to be generated as a consequence of the retrograde signaling. The rate of appearance of propagated potentiation (see Figs. 3, 5) indicates that the cytoplasmic signal is produced concurrently with the induction or expression of LTP and propagates rapidly to other synapses. The lack of potentiation at synapses onto GABAergic neurons further suggests that susceptibility to the propagating signal depends on the postsynaptic target cell. The nature of this signal remains to be determined. The rapidity of the spread of synaptic potentiation suggests that the cytoplasmic signaling may be performed by active processes, e.g., regenerative waves of second messengers such as $\mathrm{Ca}^{2+}$ and cAMP (Fitzsimonds and Poo, 1998). Alternatively, macromolecular signals, e.g., kinases, phosphatases, and GTPases, may be transported or activated by signals associated with fast axonal transport and serve to modulate secretion machinery or postsynaptic receptors throughout the neuron (Lux and Veselovsky, 1994). Colchicine-sensitive retrograde flow of nerve growth factors received at nerve terminals is known to be responsible for the maintenance of global neuronal properties, including the integrity of synapses at the dendrites (Purves, 1975). A cytoplasmic signal was also implicated in the spread of LTD (Cash et al., 1996; Fitzsimonds et al., 1997; Goda and Stevens, 1998). In these hippocampal cultures, backpropagation and presynaptic lateral propagation of LTD were observed (Fitzsimonds et al., 1997). However, unlike the input specificity found here for synapses on the postsynaptic cell, there was significant postsynaptic lateral propagation of LTD. Postsynaptic spread of LTP to a distance of $\sim 70 \mu \mathrm{m}$ from the site of induction has been observed in hippocampal slice culture (Engert and Bonhoeffer, 1997). It is not clear whether the absence of postsynaptic spread observed here in dissociated cultures is caused by more extensive washout of the extracellular factor(s) 
postulated in the latter study or by the different stimulation paradigm used in the induction of LTP. In intact CNSs, the connection between a pair of neurons consists of only a rather limited number of synaptic contacts, compared with that in the culture system. It remains to be determined whether LTP of a few synapses can generate a long-range propagating signal in the cytoplasm. On the other hand, a single high-efficacy connection observed in culture could be analogous to the in vivo situation of divergent outputs of a neuron to a group of synchronously firing postsynaptic cells, whereby propagating signals from various terminals undergoing LTP could be summated.

It remains to be determined whether potentiation at the "propagated" sites shares the same expression mechanisms with that at the induction sites. Although it is possible the apparent potentiation at the propagated sites is consistent with synaptic modification, other possibilities exist. Recent results suggest that correlated spiking in these cultures could result in changes in neuronal excitability in the presynaptic neuron, possibly because of changes in voltage-dependent $\mathrm{Na}^{+}$conductance (K. Ganguly, L. Kiss, and M.-m. Poo, unpublished observation). Such "nonspecific" changes in active conductance could contribute to the apparent induced and propagated LTP. For example, changes in excitability could affect $\mathrm{Ca}^{2+}$ influx at axonal terminals after stimulation and thus the efficacy of transmitter release, contributing to both the induction and presynaptic lateral propagation of LTP. On the other hand, if the recorded synaptic currents include active components attributable to imperfect voltage clamp, changes in active conductance at the dendrites of the presynaptic cell could also contribute to an apparent backpropagated potentiation. However, because the change in excitability could be eliminated (by selective blockade of presynaptic PKC activity) without significantly affecting the induction of LTP (Ganguly, Kiss, and Poo, unpublished observation) and because propagated LTP was similar in extent to the induced LTP, the excitability change is unlikely to account for all the changes we observed. Change in the intrinsic properties at the presynaptic cell induced by correlated activity apparently also requires retrograde as well as cytoplasmic signaling factors, which may be responsible for changes in synaptic transmission. Furthermore, with respect to neuronal integration in neural networks, changes in active conductance may have effects similar or equivalent to those of changes in synaptic transmission.

In summary, we have found a set of rules for the spread of potentiation induced by correlated presynaptic and postsynaptic excitation in a cultured neural network. These rules may be relevant for consideration of activity-induced synaptic modification at the network level and for formulation of more biologically plausible learning algorithms in neural network models (Rumelhart et al., 1986; Churchland and Sejnowski, 1992; Rolls and Treves, 1998). The existence of presynaptic lateral propagation of LTP dictates that the output synapses of a single excitatory neuron are modified as an integral unit in activity-dependent synaptic plasticity, with changes at each synapse affecting the others. Such coordinated changes among synapses may contribute to the development of synchronous firing in the cortex (Singer and Gray, 1995). Because both LTP and LTD have now been shown to spread laterally and backward in the presynaptic neuron, opposite retrograde signals received by its divergent output terminals may be effectively integrated. This presynaptic integration of plasticity may serve important roles in the development and functioning of neural circuits. Although these findings in cell cultures may represent an exaggeration of phenomena occurring in vivo, the simplicity of the culture system had allowed us to discover a novel cellular process associated with synaptic plasticity that is likely to be present to varying degrees in more complex neural systems.

\section{REFERENCES}

Arancio O, Kiebler M, Lee CJ, Lev-Ram V, Tsien RY, Kandel ER, Hawkins RD (1996) Nitric oxide acts directly in the presynaptic neuron to produce long-term potentiation in cultured hippocampal neurons. Cell 87:1025-1035.

Betz WJ, Bewick GS (1992) Optical analysis of synaptic vesicle recycling at the frog neuromuscular junction. Science 255:200-203.

Bi G-q, Poo M-m (1998) Synaptic modifications in cultured hippocampal neurons: dependence on spike timing, synaptic strength, and postsynaptic cell type. J Neurosci 18:10464-10472.

Bliss TV, Collingridge GL (1993) A synaptic model of memory: longterm potentiation in the hippocampus. Nature 361:31-39.

Bliss TV, Lømo T (1973) Long-lasting potentiation of synaptic transmission in the dentate area of the anaesthetized rabbit following stimulation of the perforant path. J Physiol (Lond) 232:331-356.

Bonhoeffer T, Staiger V, Aertsen A (1989) Synaptic plasticity in rat hippocampal slice cultures: local "Hebbian" conjunction of pre- and postsynaptic stimulation leads to distributed synaptic enhancement. Proc Natl Acad Sci USA 86:8113-8117.

Bonhoeffer T, Kossel A, Bolz J, Aertsen A (1990) Modified Hebbian rule for synaptic enhancement in the hippocampus and the visual cortex. Cold Spring Harb Symp Quant Biol 55:137-146.

Brown TH, Kairiss EW, Keenan CL (1990) Hebbian synapses: biophysical mechanisms and algorithms. Annu Rev Neurosci 13:475-511.

Cash S, Zucker RS, Poo M-m (1996) Spread of synaptic depression mediated by presynaptic cytoplasmic signaling. Science 272:998-1001.

Christie BR, Abraham WC (1992) NMDA-dependent heterosynaptic long-term depression in the dentate gyrus of anaesthetized rats. Synapse 10:1-6.

Churchland PS, Sejnowski TJ (1992) The computational brain. Cambridge, MA: MIT.

Engert F, Bonhoeffer T (1997) Synapse specificity of long-term potentiation breaks down at short distances. Nature 388:279-284.

Fitzsimonds RM, Poo M-m (1998) Retrograde signaling in the development and modification of synapses. Physiol Rev 78:143-170.

Fitzsimonds RM, Song HJ, Poo M-m (1997) Propagation of activitydependent synaptic depression in simple neural networks. Nature 388:439-448.

Goda Y, Stevens CF (1998) Readily releasable pool size changes associated with long term depression. Proc Natl Acad Sci USA 95:1283-1288.

Gustafsson B, Wigström H, Abraham WC, Huang YY (1987) Long-term potentiation in the hippocampus using depolarizing current pulses as the conditioning stimulus to single volley synaptic potentials. J Neurosci 7:774-780.

Hamill OP, Marty A, Neher E, Sakmann B, Sigworth FJ (1981) Improved patch-clamp techniques for high-resolution current recording from cells and cell-free membrane patches. Pflügers Arch 391:85-100.

Hebb DH (1949) The organization of behavior. New York: Wiley.

Katz LC, Shatz CJ (1996) Synaptic activity and the construction of cortical circuits. Science 274:1133-1138.

Kelso SR, Ganong AH, Brown TH (1986) Hebbian synapses in hippocampus. Proc Natl Acad Sci USA 83:5326-5330.

Kossel A, Bonhoeffer T, Bolz J (1990) Hebbian synapses in rat visual cortex. NeuroReport 1:115-118.

Lux HD, Veselovsky NS (1994) Glutamate-produced long-term potentiation by selective challenge of presynaptic neurons in rat hippocampal cultures. Neurosci Lett 178:231-234.

Lynch GS, Dunwiddie T, Gribkoff V (1977) Heterosynaptic depression: a postsynaptic correlate of long-term potentiation. Nature 266:737-739.

Maccaferri G, McBain CJ (1996) Long-term potentiation in distinct subtypes of hippocampal nonpyramidal neurons. J Neurosci 16:5334-5343.

Maccaferri G, Toth K, McBain CJ (1998) Target-specific expression of presynaptic mossy fiber plasticity. Science 279:1368-1370.

Magee JC, Johnston D (1997) A synaptically controlled, associative signal for Hebbian plasticity in hippocampal neurons. Science 275:209-213. 
Malenka RC, Nicoll RA (1999) Long-term potentiation-a decade of progress? Science 285:1870-1874.

Markram H, Lubke J, Frotscher M, Sakmann B (1997) Regulation of synaptic efficacy by coincidence of postsynaptic APs and EPSPs. Science 275:213-215.

McMahon LL, Kauer JA (1997) Hippocampal interneurons express a novel form of synaptic plasticity. Neuron 18:295-305.

Mermelstein PG, Haruhiko B, Deisseroth K, Tsien RW (2000) Critical dependence of cAMP response element-binding protein phosphorylation on L-type calcium channels supports a selective response to EPSPs in preference to action potentials. J Neurosci 20:266-273.

Nicoll RA, Malenka RC (1995) Contrasting properties of two forms of long-term potentiation in the hippocampus. Nature 377:115-118.

Purves D (1975) Functional and structural changes in mammalian sympathetic neurones following interruption of their axons. J Physiol (Lond) 252:429-463.

Rae J, Cooper K, Gates P, Watsky M (1991) Low access resistance perforated patch recordings using amphotericin B. J Neurosci Methods 37:15-26.

Reyes A, Lujan R, Burnashev N, Somogyi P, Sakmann B (1998) Targetcell-specific facilitation and depression in neocortical circuits. Nat Neurosci 1:279-285.

Rolls ET, Treves A (1998) Neural networks and brain function. New York: Oxford UP.
Rumelhart DE, Hinton GE, Williams RJ (1986) Learning internal representations by error propagation. In: Parallel distributed processing (Feldman JA, Hayes PJ, Rumelhart DE, eds), pp 318-362. Cambridge, MA: MIT

Ryan TA, Reuter H, Wendland B, Schweizer FE, Tsien RW, Smith SJ (1993) The kinetics of synaptic vesicle recycling measured at single presynaptic boutons. Neuron 11:713-724.

Scanziani M, Malenka RC, Nicoll RA (1996) Role of intercellular interactions in heterosynaptic long-term depression. Nature 380:446-450.

Schuman EM, Madison DV (1994) Locally distributed synaptic potentiation in the hippocampus. Science 263:532-536.

Singer W, Gray CM (1995) Visual feature integration and the temporal correlation hypothesis. Annu Rev Neurosci 18:555-586.

Wang X-h, Poo M-m (1997) Potentiation of developing synapses by postsynaptic release of neurotrophin-4. Neuron 19:825-835.

Wilcox KS, Buchhalter J, Dichter MA (1994) Properties of inhibitory and excitatory synapses between hippocampal neurons in very low density cultures. Synapse 18:128-151.

Williams JH, Errington ML, Lynch MA, Bliss TV (1989) Arachidonic acid induces a long-term activity-dependent enhancement of synaptic transmission in the hippocampus. Nature 341:739-742.

Zhang LI, Tao HW, Holt CE, Harris WA, Poo M-m (1998) A critical window for cooperation and competition among developing retinotectal synapses. Nature 395:37-44. 